Pain Physician. 2003;6:269-271, ISSN 1533-3159

An Original Contribution

\title{
Can the Sacroiliac Joint Cause Sciatica?
}

\author{
Joseph D. Fortin, DO*, Joel A. Vilensky, PhD\#, and Glenn J. Merkel, PhD\#
}

\begin{abstract}
In this brief study we provide evidence that earlier and more recent findings pertaining to the anatomy and physiology of the sacroiliac joint suggest that dysfunction in this
\end{abstract}

Prior to 1934, Sacroiliac joint (SIJ) dysfunction was considered to be the most likely source of idiopathic low back pain and sciatica $(1,2)$. In that year, Mixter and Barr (3) suggested in their classic report that most low back pain could be attributed to the herniation of an intervertebral disc. However, disc pathology does not account for all low back pain (47) and SIJ dysfunction is again being recognized as a source of low back pain in some patients (8-12).

To help differentiate SIJ dysfunction from other low back pain conditions, one of us (JDF) defined pain referral maps based upon stimulation of the SIJ capsule of ten normal subjects by direct intra-articular injection of contrast medium and local anesthetic (8). The experiment revealed a common area of hypesthesia in the buttocks running approximately $10 \mathrm{~cm}$ inferiorly and $3 \mathrm{~cm}$ laterally from the posterior superior iliac spine. These findings were considered consistent with sensory changes in the area of supply of the S1-2 (S3) dorsal rami (11).

Whereas the Fortin et al's $(8,9)$ studies demonstrated that SIJ dysfunction may cause low back or hip pain, they did not present any evidence that sciatica may be related to this condition. However, more recent studies on contrast extravasation during SIJ arthrography (10) and

From *Spine Technology and Rehabilitation, Fort Wayne, IN, and Indiana University School of Medicine, Fort Wayne, IN, and \# Indiana University School of Medicine, Fort Wayne, IN. Address Correspondence: Joseph D. Fortin, DO, Spine Technology and Rehabilitation, 7230 Engle Road, Suite 210, Fort Wayne, IN 46804. Email: fortin@pol.net

Funding: There was no external funding in preparation of this manuscript. joint could, similar to a herniated lumbar disc, produce pain along the sciatic nerve. These observations might explain some of the cases of sciatica in which no disc pathol- ogy can be found.

Keywords: Sacroiliac Joint dysfunction, intervertebral disc, Substance P

SIJ innervation $(11,12)$ suggest that there are anatomical and physiological reasons to believe that SIJ dysfunction can lead to symptoms that resemble classic sciati$\mathrm{ca}$, and which are usually considered consistent with disc pathology. This observation could explain the lack of disc pathology in some patients with these conditions $(4,8)$.

The present investigation sought to determine if symptomatic SIJs contained neurotransmitters and if there exists an anatomic pathway for them to transmit pain signals to elements of the sciatic nerve.

\section{Methods}

To determine if the sacroiliac joint is a putative cause of sciatica, two models were developed: 1) a structural model to determine if there is an anatomical pathway for the sacroiliac joint to communicate with elements of the sciatic nerve; and 2) a physiological one to investigate a mechanism for transmission of pain from the SIJ to the sciatic nerve.

\section{Phase 1: The Anatomical Model}

In phase one of this design, Fortin et al (10) examined 43 patients with low back pain for greater than two months duration. SIJ arthrograms were obtained in all patients (31 had bilateral procedures). After the injection of contrast medium, anterior-posterior, lateral and oblique postarthrographic CT scans were also obtained for the same joints.

\section{Phase 2: The Physiological Model}

Two tissue samples were analyzed from patients, ages 31-55 years of age, undergoing sacroiliac arthrodesis for intrac- table, unilateral SIJ pain. The criteria included $90 \%$ or greater pain relief from two image-guided anesthetic injections. Patients with symptomatic discs, posterior joints, radiculopathies or spinal stenosis were excluded. The operation involved a posterior approach to SIJ arthrodesis with internal fixation. Posterior ligamentous samples were extirpated adjacent to the medial aspect of the posterior superior iliac spine and the sacrum en bloc using Bovie electrocoagulation sub-periosteally on the joint surfaces. The tissue was then subjected to a neurohistologic and immunohistochemical investigation of the dorsal SIJ ligament, as previously detailed, with the following modifications specific to staining for substance P: The tissue was fixed with 5\% acetic acid in 95\% ethanol instead of paraformaldehyde and endogenous peroxidase activity was quenched by treating sections with $0.3 \%$ hydrogen peroxide for fifteen minutes instead of overnight. Also, the anti-substance $\mathrm{P}$ antibody was diluted 1:250 instead of 1:350.

\section{RESULTS}

\section{Phase 1 - The Anatomical Model}

Sixty-one percent of all SIJs showed extravasation of contrast medium. Of five patterns revealed, three represented potential pathways between the SIJ and surrounding neural structures. Sixteen percent (12 cases) of the SIJs showed a ventral extravasation near the lumbosacral plexus. Thirty-two percent (24 cases) of the SIJs revealed dorsal leakage. In six of these cases ( $8 \%)$, contrast medium was seen to enter the $\mathrm{S} 1$ dorsal foramen. Lastly, 3\% (2 cases) revealed contrast medium flowing superiorly reaching the L5 root 
canal anteromedially.

Phase 2 - The Physiological Model

Our improved histochemical staining technique for Substance $\mathrm{P}$ allowed us to disclose Substance P readily in most portions of the samples we investigated, including the isolated intraligamentous deposition of Substance P (Figure 1B) and the presence of Substance $\mathrm{P}$ in what our likely articular receptors (Figure 1C and 1D).

\section{DISCUSSION}

We have previously demonstrated that the periarticular tissues of the human
SIJ contain mechanoreceptors, nerves and nerve fascicles presumably designed to convey proprioceptive and pain impulses to the central nervous system (12). However, in that study of the histology of periarticular tissues removed from patients undergoing sacroiliac arthrodeses for intractable pain, we were unable to demonstrate the presence of the neurotransmitter, Substance P, which has repeatedly been associated with transmission of pain and "neurogenic inflammation" (13). Using our improved technique has now definitively revealed the presence of Substance $\mathrm{P}$ in the periarticular tissues of the SIJs of these patients (Figure 1). We have identified both undifferentiated deposition of Substance P (B) and what appear to be articular receptors that contain this neuropeptide (C\&D). This observation taken with those from the extravasation study (10) provides a scenario whereby SIJ dysfunction could manifest as sciatica-like symptoms. That is, in a traumatized and inflamed joint, extravasation of synovial fluid containing inflammatory mediators including Substance P could traverse any of the three pathways described and irritate one or more of the neural elements that compose the sciatic nerve (L4-S2) (3).

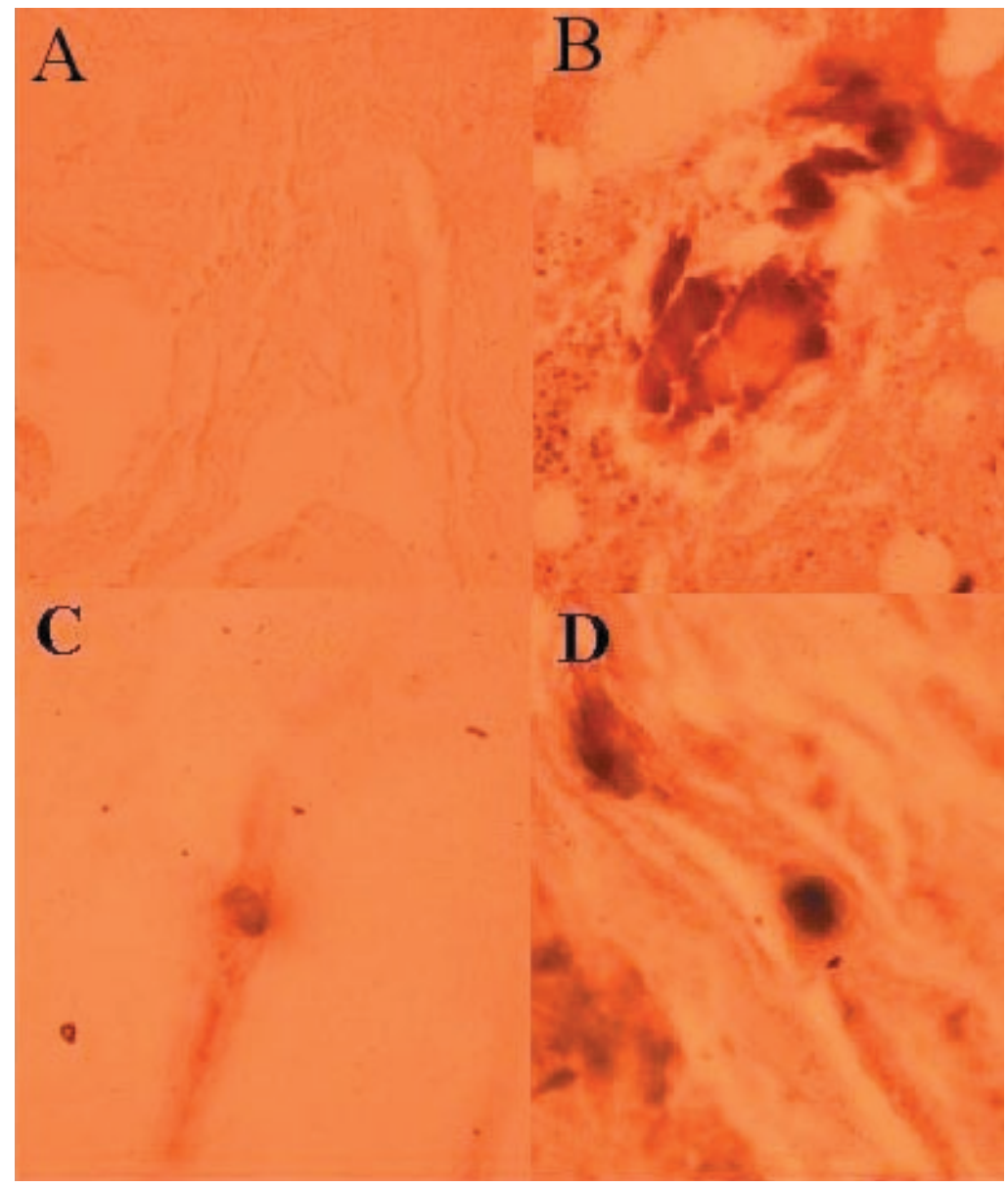

Figure 1. Substance P labeled-periarticular tissue from human SIJs. A: Tissue used for control (without antiSubstance $P$ antibody) showing the absence of any dark staining that would indicate the presence of Substance P. Mag:original X40. B: Large deposition of Substance P in adipose-type SIJ tissue. Mag:original X80. C. Same patient as $A$ and $B$ showing central Substance P-labeled structure, possibly an articular receptor for pain or pressure. Mag:orignal X40. D. Different patient than in A, B and C showing similar Substance P-labeled articular structure. Mag:original $X 40$. 


\section{Conclusion}

Low back pain and sciatica continues to be an enigmatic condition for many patients, and a frustrating one for their physicians. Certainly, discogenic origins accounts for some, but not all, of the pain and discomfort associated with these conditions. Thus, we suggest here that the pre-1934 belief that sciatica may also be associated with SIJ dysfunction has merit. The mechanism by which such dysfunction could produce sciatica was unknown at that time. Here, we present a model based on observed joint extravasation patterns and the demonstrated presence in the joint of the pain neurotransmitter, Substance P, which may account for sciatic stmptoms in patients with SIJ dysfunction.

\author{
Author Affiliation: \\ Joseph D. Fortin, DO \\ Medical Director \\ Spine Technology and Rehabilitation \\ Clinical Professor \\ Indiana University School of Medicine \\ 7230 Engle Road, Suite 210 \\ Fort Wayne, IN 46804 \\ E-mail: fortin@pol.net \\ Joel A. Vilensky, PhD \\ Professor of Anatomy and Cell Biology \\ Indiana University School of Medicine \\ 2101 Coliseum Blvd, East \\ Fort Wayne, IN 46805 \\ Email: vilensk@ipfw.edu \\ Glenn J. Merkel, PhD \\ Professor of Microbiology and \\ Immunology \\ Indiana University School of Medicine \\ Fort Wayne, IN 46805 \\ Email: merkel@ipfw.edu
}

\section{REFERENCES}

1. Goldthwait GE, Osgood RB. Consideration of the pelvic articulations from an anatomical, pathological, and chemical standpoint. Boston Med Surg / 1905; 152:593-601.

2. Brooke R. The sacroiliac joint. J Anat 1924; 58:299-305.

3. Mixter WJ, Barr JS. Rupture of the intervertebral disc with involvement of the spinal canal. N Engl / Med 1934; 211:210-215.

4. Burton CV, Kirkaldy-Willis WH, Yong-Hing $\mathrm{K}$ et al. Causes of failure of surgery on the lumbar spine. Clin Orthop 1981; 157:191-199.

5. Bodin SD, David DO, Dina TS. Abnormal magnetic resonance scans of the lumbar spine in asymptomatic subjects. A prospective investigation. I Bone Joint Surg (Am) 1990; 72:403-408.

6. Hitselberger WE, Witten RM. Abnormal myelograms in asymptomatic patients. J Neurosurg 1968; 28:204-206.

7. Jensen MC, Brant-Zawadski MN, Obuchowski $\mathrm{N}$ et al. Magnetic resonance imaging of the lumbar spine in people without back pain. N Engl J Med 1994; 2:69-73.
8. Fortin JD, Dwyer AP, West S, et al. Sacroiliac joint: Pain referral maps upon applying a new injection/arthrography technique. Part I: Asymptomatic volunteers. Spine 1994; 19:1475-1482.

9. Fortin JD, Aprill CN, Ponthieux B et al. Sacroiliac joint: Pain referral maps upon applying a new injection/arthrography technique. Part II: Clinical evaluation. Spine 1994; 19:1483-1489.

10. Fortin JD, Washington WJ, Falco FJE. Three pathways between the sacroiliac joint and neural structures. Am J Neuroradiol 1999; 20:1429-1434.

11. Fortin JD, Kissling RO, O'Connor BL, et al. Sacroiliac joint innervation and pain. Am J Orthoped 1999; 38:687-690.

12. Vilensky JA, O'Connor BL, Fortin JD, et al. Histologic analysis of neural elements in the human sacroiliac joint. Spine 2002; 27:1202-1207.

13. Vilensky JA. Innervation of the joint and its role in osteoarthritis. In: Brandt KD, Doherty M, Lohmander LS (eds) Osteoarthritis, Oxford University Press, Oxford, 2003 (in press). 